\title{
The Application of Traditional Window Lattice Ornamentation in Modern Interior Design
}

\begin{abstract}
Xia Wang ${ }^{1, *}$
${ }^{1}$ Hainan Vocational University of Science and Technology, Haikou, Hainan 571158, China

"Corresponding author. Email: wangxia20202020@163.com

ABSTRACT

The window lattice ornamentation in traditional Chinese architecture is the cultural accumulation of China's five thousand years of development, and the crystallization of the wisdom of the Chinese people. Traditional window lattice ornamentation contains its unique cultural meaning. In the continuous development of China, window lattice ornamentation has been passed down and has been used in modern interior design. Through the quotation and refinement of traditional window lattice ornamentation, the designer should not only satisfy people's requirements for indoor space functions, but also improve the cultural flavor of indoor space. This article introduces traditional window lattice ornamentation based on relevant documents and materials. The traditional window lattice ornamentation is made up of three types of patterns, namely, crutch pattern, horizontal and vertical rib pattern, and rhombus pattern. Through specific analysis of window lattice ornamentation structure design, it shows the wisdom of Chinese craftsmen in window lattice construction. Combined with the application of window lattice ornamentation in modern interior design, it demonstrates the spatial function of traditional window lattice patterns in modern interiors, and at the same time improves the aesthetics and cultural charm of modern indoor spaces.
\end{abstract}

Keywords: traditional window lattice ornamentation, modern times, interior design, application

\section{INTRODUCTION}

As it known to all, China is one of the four ancient civilizations. China has formed an architectural culture with Chinese characteristics in the long-term development. Window is an important part of traditional wooden structure building, it is people's visual center, aesthetic center, and the focus of traditional building decoration [1]. Traditional window lattice ornamentation contains its unique cultural connotation, has research value, and can provide design inspiration for modern designers.

\section{CONCEPT AND PATTERN STRUCTURE OF TRADITIONAL WINDOW LATTICE ORNAMENTATION}

\section{A. The concept of window lattice ornamentation}

The window lattice is the window pane, which is an important part of the traditional wooden building and the aesthetic center of the building. The window lattice ornamentation embodies the wisdom of the Chinese sons and daughters, shows the folk customs and customs of various places, and expresses people's expectations for a better life.

\section{B. The pattern structure of traditional window lattice ornamentation}

1) Crutch pattern: The main styles of this pattern are lantern frame pattern, ఔ pattern, panchang pattern, etc. The window lattice with the pattern of the abdomen will have a hollow in the middle part, and the pattern or window paper will be pasted on this part, giving people a beautiful feeling [2]. First, it is the lantern frame pattern. This kind of pattern is a pattern composed of fringe strips. The openwork cluster flowers and kazi flowers are integrated into the fringe strips, which contain hope for a bright future and are relatively practical. Compared with other patterns, the lantern frame is provided with a large cavity, which is beneficial to improve indoor lighting and ventilation effects. Second, it is the panchang pattern. This kind of pattern is one of the eight treasures of Buddhism, and it contains the hope of all things being satisfactory. The panchang pattern is the use of lattice bars to form a reciprocating pattern, which has a continuous meaning.

2) Horizontal and vertical rib pattern: This pattern is a pattern composed of lattice strips in the same format, and its main styles are 回-shaped, diagonal grid, tofu block, etc. The fringe used in this pattern is 
narrower and thicker, and its three-dimensional effect is better [3]. When passing through the horizontal and vertical ribs, there will be a feeling of changing patterns. The horizontal and vertical rib pattern is flexible, dignified and elegant. There are two types of horizontal and vertical rib pattern commonly used in architecture: First, it is the Bubujin pattern. This pattern is a pattern formed by using short strips according to the laws of 工-shaped, lying silkworm, etc. It means a bright future and has a wide range of applications. Second, it is the ice crack pattern. This pattern is made up of small lattice strips through the method of arranging a bucket. It has the meaning of cold windows and was often used in private school buildings in ancient times.

3) Rhombus pattern: This pattern is developed from the mullion mesh fabric. The mullions used in the pattern are all at a $60^{\circ}$ angle. Although this pattern is relatively simple, its variations are richer, and it is widely used in architecture [4]. The rhombus pattern commonly used in architecture is as follows: First, it is double cross four parts rhombus pattern. This pattern can be divided into orthogonal and oblique. Orthogonal refers to the lattice bar presents a $90^{\circ}$ angle, the window frame is parallel to the intersection line, the horizontal line and the horizontal frame line are parallel, the vertical line and the vertical frame line are parallel; the diagonal lattice bar presents a $90^{\circ}$ angle, but it is at a $45^{\circ}$ angle to the frame. Second, it is the triple cross six parts rhombus pattern. In this pattern, there is only one mullion in an orthogonal state, and it is vertical up and down, and both the mullion and the mullion present a $60^{\circ}$ angle with the mullion. In this pattern, only one mullion in a diagonal state is horizontal, and the other mullions are at an angle of $60^{\circ}$ with this mullion.

\section{STRUCTURAL DESIGN OF TRADITIONAL WINDOW LATTICE ORNAMENTATION}

\section{A. Form structure design}

The beauty of the form of traditional window lattice patterns in China is displayed on multiple levels, which mainly involve: the shape of the window sash, the center of the window sash, the composition ratio of the sash ring sash, and the contrast between the real and the virtual of the lattice carvings. The window is an important part of the whole building. The first thing people see when they see the building is the shape and size of the window. The length and width ratio of rectangular windows is not fixed; when designing floorto-ceiling partition windows, it is necessary to ensure the rationality of the height and width ratio of column partitions. Affected by external factors, the height and width ratios of window sashes in different dynasties are different.

In the whole window, the lattice heart is the key point of window decoration, and the hollow lattice is usually used [5]. Decorative patterns are not required for skirtboards, and even there are decorative patterns, they are relatively simple. Most of the upper and lower ribbon ring plates are decorated with embossed and engraved carvings, and their decorations are coordinated with the grid. This not only satisfies the functional requirements of air circulation and viewing of the partition, but also improves the aesthetics of the partition.

\section{B. Space structure design}

The lattice center of the window is at the center of the entire window, which occupies a large area in the entire window. At the same time, the grid is also an important structure that affects the ventilation and lighting effects of windows, and it is a structure that affects the decorative effect of the entire window. Under normal circumstances, the lattice center is made as a straight line or a curve through the hollow lattice, and some window lattice centers are provided with carvings at the node positions to enhance the aesthetics of the lattice center. Part of the window grid is provided with embossed flower panels in the middle position, thus forming the icy crack pattern 寿 character, cross brocade group 寿 and other carved carvings. Relief imitating landscape paintings, relief character stories, etc., are all multi-level superimposed compositions, with rich levels that can fully show the beauty of space.

Windows not only have a decorative effect, they also have the function of distinguishing indoor and outdoor spaces. The lattices and carvings used in leaky windows and opening windows can enhance the partitioning effect of building space and enhance the aesthetics of building space. Leaky windows are an important structure that separates the indoor and outdoor spaces. When people look from the outside to the inside, they can feel the beauty of the hollow flower window; when they look from the inside to the outside, the exterior scenery is faintly visible. With the development of technology, the combination of glass and windows can enhance the modernity of leaky windows.

\section{Color structure design}

The windows in traditional buildings in China are rich in colors, with regional and custom characteristics. Most windows in royal buildings choose red, green, and gold, which can show the majesty of imperial power. Jiangnan buildings are characterized by elegance and simplicity, and most of their windows are made of clear-white wood. Most of the windows in the temple are decorated with golden yellow patterns, which can 
reflect the solemnity of the temple. In short, color is of great significance in traditional lattice ornamentation. Different colors, different compositions, and different types of windows can show different aesthetics.

\section{Moral structure design}

Different traditional lattice patterns in China have different meanings, and each pattern expresses people's expectations for a better life. The combination of different patterns also expresses different intentions, and people can get a sense of beauty through these patterns. Under normal circumstances, the traditional window lattice patterns mainly contain the meanings of "blessing, fortune, longevity, happiness and wealth".

1) Praying for blessing: The combination of stone and fish in the traditional window lattice pattern contains the expectation of "more than good luck"; the combination of three sheep in the traditional window lattice pattern contains the meaning of "three sheep start the peace"; the combination of lily, Ganoderma lucidum and persimmon or lion in the traditional window lattice pattern implies the meaning of "all goes well"; the combination of four bats in the traditional window lattice pattern implies the meaning of "welcoming good luck and happiness".

2) Praying for fortune: The combination of a vase and three weapon halberds in the traditional window lattice pattern contains the meaning of "leveling up to three levels"; the combination of a carp and a dragon gate in the traditional window lattice pattern contains the meaning of "being among the best of candidates"; the combination of cranes and turtles in the traditional window lattice pattern contains the meaning of "being the only one of the top".

3) Praying for longevity: The combination of five bats in the traditional window lattice pattern contains the meaning of "five bats blessing for longevity"; the combination of tortoise, peony and 寿 character in the traditional window lattice pattern contains the meaning of "long life and wealth".

4) Praying for happiness: The combination of the character "䱏" and two magpies in the traditional window lattice pattern implies "double blessing"; the combination of China rose flowers and magpies in the traditional window lattice pattern implies "four seasons happiness".

5) Praying for wealth: The combination of cornucopia and copper coins in the traditional window lattice pattern implies the meaning of "adding wealth and treasure"; the combination of goldfish and aquatic plants in the traditional window lattice pattern implies the meaning of "prosperity and wealth"; the combination of fish and peony in the traditional window lattice pattern contains the meaning of "more than wealth".

Everyone has the psychology of seeking advantages and avoiding disadvantages. The use of auspicious patterns in traditional window lattice patterns can fully show people's expectations for a better life, and it can bring people spiritual comfort. Traditional window lattice pattern are the yearning and sustenance of people, which can give people a beautiful feeling while meeting the functional requirements of windows.

\section{THE APPLICATION OF TRADITIONAL WINDOW LATTICE ORNAMENTATION IN MODERN INTERIOR DESIGN}

While China's economic and cultural exchanges with other countries are increasing, Chinese traditional culture has been impacted and influenced by foreign cultures from the west, which arouses people's thinking and attention to traditional culture. The application and transformation of traditional consciousness based on modern aesthetics can give full play to its role and enhance the vitality of traditional culture. The application of China's traditional window lattice patterns in modern interior design can fully demonstrate the cultural characteristics of the times.

\section{A. The application of traditional window lattice ornamentation in modern patterns}

In recent years, more and more people like to incorporate my Chinese traditional culture and style into modern interior space design, and traditional window lattice ornamentation is the most widely used pattern [6]. In modern interior space design, most of the traditional window lattice patterns are used on the top surface, wall surface, and partitions. Most of the patterns selected are 万 pattern, 回 pattern, and moire pattern. Patterns such as dragon and phoenix patterns are used in some furniture. However, this method of directly using traditional window lattice ornamentation cannot be used casually. In the application process, designers need to fully analyze the entire indoor space style, environment, regional culture, and folk customs, and choose traditional window lattice ornamentation that can show the characteristics of the day. At the same time, designers need to ensure the rationality of the selection of traditional window lattice ornamentation, ensure that the traditional window lattice ornamentation has outstanding characteristics, and display the cultural characteristics of the indoor space while giving full play to their decorative functions.

\section{B. Refining and application of traditional window lattice ornamentation in modern}

When using this method to apply traditional window lattice ornamentation, designers need to 
simplify and organize the traditional window lattice ornamentation to ensure that the traditional window lattice ornamentation is suitable for modern indoor spaces. Normally, designers can adopt the following methods when simplifying traditional window lattice ornamentation: First, they can make the simplification from the perspective of artistic conception. This requires designers to in-depth study and understand the meaning and implication of traditional window lattice patterns. According to the characteristics of the indoor space, the "meaning" in the traditional window lattice pattern need to be extracted. Second, designers can make the simplication from the perspective of form. Designers need to simplify and generalize the forms of traditional window lattice patterns, and then use them in modern interior space design to ensure that modern indoor spaces have the characteristics of traditional lattice patterns, but cannot directly copy. The designer needs to understand the charm and specificity of the original traditional window lattice pattern, and simplify it to ensure that the processed window lattice pattern is suitable for modern indoor spaces. For example, in the design process of some Chinese-style study rooms, the simplified and refined window lattice patterns can be used on the bookshelves. In this way, the cultural heritage of bookshelves and modern indoor spaces can be enhanced.

\section{The application of traditional window lattice ornamentation in modern reconstruction}

When applying traditional lattice patterns in this way, designers need to split the symbols that constitute the traditional lattice patterns, and then rearrange these lattice pattern symbols through deformation, reconstruction, etc. For example, when using traditional window lattice patterns in screen design, designers can remove the middle pattern of the screen and use glass in this part, which can fully demonstrate the characteristics of traditional Chinese culture while enhancing the modern sense of indoor space. Some traditional window lattice patterns are inconsistent with today's aesthetic concepts, so designers can optimize the structure of such traditional window lattice patterns, or use only part of the characteristics of traditional window lattice patterns to transform them into new patterns. While people understand and master traditional culture, they can also innovate traditional culture. In this link, some prejudices will inevitably arise. It is a must to correct and eliminate these prejudices, innovate traditional culture, and form a new culture. When the French architect and designer Paul Andrew designed the interior space of the National Grand Theater of China, he set up a red curve decoration, which is formed by hanging steel cables from the top of the building to form a semi-ceiling structure. This shape is a modification of the traditional moiré pattern. The application of this pattern can enhance the beauty and cultural heritage of the entire space.

\section{CONCLUSION}

During the five thousand years of development in China, a traditional culture with Chinese characteristics has been formed. Traditional window lattice ornamentation is one of the components of traditional culture. After a long period of accumulation and innovation, the current lattice ornamentation has been formed. At present, the value of traditional window lattice ornamentation is not only ornamentation, but a rich culture. The use of traditional window lattice ornamentation in modern interior space design can enhance the aesthetics of the interior space. At the same time, the traditional window lattice ornamentation used based on modern aesthetic concepts, and the use of new materials and new technologies can enhance the modernity and cultural characteristics of the traditional window lattice ornamentation. The designers cannot simply copy and apply traditional window lattice patterns, lest the traditional lattice patterns do not meet the requirements of modern interior space design. It is necessary to adopt simplification, refinement, and reorganization methods to process traditional window lattice patterns, so as to ensure the functional requirements of modern indoor space, while improving the aesthetics and cultural charm of modern indoor space.

\section{References}

[1] Zhan Qinchuan, An Qiang, On the Application of Traditional Stone Carving in Modern Interior Design [J]. Appreciation, 2018, 000(06X): P.292-293. (in Chinese)

[2] Sheng Jiayi, The Application of Chinese Traditional Cultura Elements in Modern Interior Design [J]. Appreciation, 2018, 000(014): P.243-244. (in Chinese)

[3] Hu Yajing, The Use of Chinese Traditional Cultural Elements in Modern Interior Design [J]. Construction Materials \& Decoration, 2019, 000(011): 74-75. (in Chinese)

[4] Tian Chenglong, On the Application of Zhuang Nationality's Traditional Cultural Elements in Modern Interior Design [J]. Art Science and Technology, 2019, 032(004): 69. (in Chinese)

[5] Yang Zhiwu, The Application of Traditional Decoration Patterns in Modern Interior Design [J]. Journal of Heihe University, 2018, 9(1): 171-172. (in Chinese)

[6] Cheng $\mathrm{Yu}$, Application of Chinese Traditional Culture in Modern Interior Design [J]. Decoration Refurbishment Chntre, 2019, 000(001): 13. (in Chinese) 